\title{
Irinotecan, leucovorin and 5-fluorouracil (modified FOLFIRI) as salvage chemotherapy for frail or elderly patients with advanced gastric cancer
}

\author{
JUNG HAN KIM ${ }^{1}$, HYEONG SU KIM ${ }^{1}$, A RUM HAN ${ }^{1}$, IN HO MOH ${ }^{1}$, DOO CHEOL CHUNG ${ }^{1}$, DAE RO CHOI ${ }^{1}$, \\ HYUN JOO JANG ${ }^{1}$, JIN BAE KIM ${ }^{1}$, DAE HYUN YANG ${ }^{2}$, SOON IL LEE ${ }^{3}$ and DAE YOUNG ZANG ${ }^{1}$ \\ Departments of ${ }^{1}$ Internal Medicine and ${ }^{2}$ Surgery, Hallym Medical Center, Kangnam Sacred Heart Hospital, \\ Hallym University College of Medicine, Seoul 150-950; ${ }^{3}$ Department of Hemato-Oncology, Dankook University Hospital, \\ Dankook University College of Medicine, Choenan 330-715, Republic of Korea
}

Received March 8, 2012; Accepted June 28, 2012

DOI: $10.3892 / \mathrm{ol} .2012 .782$

\begin{abstract}
We retrospectively evaluated the efficacy and safety of the modified FOLFIRI regimen in frail or elderly patients with advanced gastric cancer (AGC). We reviewed 24 frail [Eastern Cooperative Oncology Group performance status (ECOG PS) of 2] or elderly (65 years or over) patients with AGC who received the modified FOLFIRI regimen as salvage chemotherapy. Patients received irinotecan $150 \mathrm{mg} / \mathrm{m}^{2}$ and leucovorin (LV) $100 \mathrm{mg} / \mathrm{m}^{2}$ as a $2 \mathrm{~h}$ intravenous infusion, followed by 5 -fluorouracil $(5-\mathrm{FU}) 2,000 \mathrm{mg} / \mathrm{m}^{2}$ as a $46 \mathrm{~h}$ continuous infusion. Among the 24 patients, $18(75 \%)$ had an ECOG PS of 2, and 11 (45.8\%) were aged 65 years or over. A total of 113 cycles were conducted, with a median number of 4 cycles per patient. A total of 3 patients achieved partial response (PR) and 8 demonstrated stable disease (SD). On an intent-to-treat basis, the overall response rate (RR) was $12.5 \%$ and the disease control rate (PR and SD) was $45.8 \%$. The median time to progression (TTP) was 2 months [95\% confidence interval (CI), 1.9-2.1 months] and the median overall survival (OS) was 5.4 months (95\% CI, 4.1-6.7 months). Grade 3-4 hematological toxicities, including neutropenia, anemia and thrombocytopenia, were observed in $6(25 \%)$, $4(16.7 \%)$ and $1(4.2 \%)$ patients, respectively. Additionally, $3(12.5 \%)$ patients developed febrile neutropenia, of which 1 succumbed to pneumonia. Grade 3-4 gastrointestinal toxicities, including nausea, vomiting, diarrhea and mucositis, were observed in $3(12.5 \%), 2(8.3 \%), 1(4.2 \%)$ and $1(4.2 \%)$ patients, respectively. In conclusion, the modified FOLFIRI regimen as
\end{abstract}

Correspondence to: Dr Jung Han Kim, Department of Internal Medicine, Hallym Medical Center, Kangnam Sacred Heart Hospital, Hallym University College of Medicine, 948-1, Daelim-1-Dong, Youngdeungpo-Gu, Seoul 150-950, Republic of Korea

E-mail: harricil@hotmail.com

Key words: advanced gastric cancer, FOLFIRI, elderly, poor performance status salvage chemotherapy for AGC patients over 65 years of age or with a poor PS was effective and acceptable. These results suggest that this regimen may be an effective option for frail or elderly patients with AGC.

\section{Introduction}

With regard to incidence and mortality, gastric cancer is one of the most common types of cancer worldwide. In Korea, gastric cancer is the most commonly occurring malignancy, accounting for approximately $15 \%$ of newly diagnosed cancer cases (1). Although its incidence in individuals younger than 50 years of age has recently increased, gastric cancer develops more frequently among patients in their seventh or eighth decade of life. To date, the only potentially curative treatment is surgery; however, a considerable number of patients present with advanced stages of the disease at the time of diagnosis. Moreover, more than half of the patients treated by complete resection eventually develop recurrent disease $(2,3)$. Palliative chemotherapy in the first- and second-line setting improves survival in advanced gastric cancer (AGC) compared to the best supportive care $(4,5)$; however, the prognosis of the advanced and recurrent disease remains poor.

Irinotecan with infusional leucovorin (LV) and 5-fluorouracil (5-FU) (FOLFIRI regimen) is widely used as a first-line treatment for patients with advanced colorectal cancer (CRC) (6). Certain studies have also identified the activity and tolerability of FOLFIRI regimens as first-line chemotherapy in patients with AGC $(7,8)$. The reported objective response rate (RR) was approximately $40 \%$, with a median overall survival (OS) of 10-12 months. Phase II studies using FOLFIRI regimens as salvage therapy for patients with AGC have demonstrated an RR of approximately $10 \%$, with a median OS of approximately 6 months (9-11). In terms of toxicity, FOLFIRI regimens have been well-tolerated.

Since FOLFIRI regimens have demonstrated an acceptable safety profile with a beneficial activity profile for patients with AGC (7-11), we used the modified FOLFIRI regimen (biweekly irinotecan $150 \mathrm{mg} / \mathrm{m}^{2}$ with $\mathrm{LV} 100 \mathrm{mg} / \mathrm{m}^{2}$ and 
5 -FU $2000 \mathrm{mg} / \mathrm{m}^{2}$ ) as salvage chemotherapy for elderly or frail patients with AGC.

We retrospectively reviewed the efficacy and safety of the modified FOLFIRI regimen as second-line chemotherapy in AGC patients in old age or with a poor performance status (PS).

\section{Patients and methods}

Patients. Between January 2005 and August 2011, 24 patients who received systemic chemotherapy with the FOLFIRI regimen as second-line chemotherapy for AGC were retrospectively analyzed. To be eligible for this study, patients had to have histologically confirmed adenocarcinoma of the stomach and unresectable or metastatic disease with at least 1 measurable lesion. Patients with a European Clinical Oncology Group performance status (ECOG PS) of 2 were eligible regardless of their age. Among the patients with an ECOG PS of 1, only those aged 65 years or over were included in the study. Additionally, patients had to fulfill the following criteria: no central nervous system metastases, no active infection, no history of other malignancies and sufficient hepatic, renal and bone marrow functions. Patients previously treated with adjuvant chemotherapy were also included. The study was approved by the Institutional Review Board (IRB) of Hallym Medical Center, Kangnam Sacred Heart Hospital, Hallym University College of Medicine, Seoul, South Korea, and as this retrospective study involved no risk to the patients, the waiver of informed consent was allowed by the IRB.

Treatment schedule. All 24 patients received irinotecan $150 \mathrm{mg} / \mathrm{m}^{2}$ and $\mathrm{LV} 100 \mathrm{mg} / \mathrm{m}^{2}$ as a $2 \mathrm{~h}$ intravenous infusion, followed by 5 -FU $2,000 \mathrm{mg} / \mathrm{m}^{2}$ as a $46 \mathrm{~h}$ continuous infusion. Antiemetic prophylaxis with $5-\mathrm{HT}_{3}$ antagonists were administered prior to irinotecan infusion, but corticosteroids were not routinely administered. Unless there was no evidence of disease progression or the patient experienced unacceptable toxicity, this regimen was repeated every 14 days to the maximum of 12 cycles.

Treatment was delayed for up to 2 weeks until patients recovered from adverse effects, the absolute granulocyte count exceeded 1,000/ $\mu 1$ and/or the platelet count exceeded $100,000 / \mu 1$. Dose reductions were made according to the highest grade of toxicity that had occurred during the previous cycle. The dose of irinotecan and 5-FU was reduced by $25 \%$ for hematological (neutropenia and thrombocytopenia) or non-hematological toxicities (mucositis and diarrhea) of grade $3-4$.

Response and toxicity evaluation. Baseline evaluation included physical examination, complete blood counts (CBC), blood chemistries and radiology examinations. Physical examination, $\mathrm{CBC}$ and blood chemistry were performed prior to each cycle. Tumor assessments by CT scans were repeated every 4 cycles. The response to the modified FOLFIRI regimen was assessed according to the guidelines of the Response Evaluation Criteria in Solid Tumors (RECIST) committee. Complete response (CR) was defined as the disappearance of all evidence of the disease, and partial response (PR) was defined as a reduction in the unidimensional tumor measurements by at least $30 \%$, without the formation of a new lesion or the progression of an existing lesion. Progressive disease (PD) was defined as an increase in the sum of the products of all measurable lesions by at least $20 \%$ or the appearance of a new lesion, including the reappearance of any lesion that previously disappeared. Stable disease (SD) was defined as a tumor response that did not fulfill the criteria for CR, PR or PD.

Toxicities were recorded according to the National Cancer Institute Common Toxicity Criteria (NCI-CTC) version 3.0. For toxicity analysis, the highest grade of toxicity in all cycles of chemotherapy was used for each patient.

Statistical analysis. Response and toxicity data were analyzed using simple descriptive statistics. Time to progression (TTP) and OS were determined from the first day of chemotherapy using the modified FOLFIRI regimen. TTP was calculated until tumor progression or mortality, and OS was calculated until the date of mortality or the last follow-up. Survival curves were established using the Kaplan-Meier method.

\section{Results}

Patient characteristics. A total of 24 patients were included in the study. Their baseline characteristics are summarized in Table I. The patients consisted of 17 males (70.8\%) and 7 females with a median age of 60.5 years (range, $30-83$ years). A total of 11 patients $(45.8 \%)$ were aged 65 years or over and $18(75 \%)$ had an ECOG PS of 2. Amongst the 24 patients, $21(87.5 \%)$ had adenocarcinoma and the remaining 3 had signet ring cell carcinoma. The most common metastatic site was the peritoneum (54.2\%), followed by the abdominal lymph node (50\%), liver (41.6\%) and lung (16.7\%). A total of $17(70.8 \%)$ patients had metastatic diseases and the remaining 7 had recurrent disease following curative resection. As first-line chemotherapy, 14 patients $(58.3 \%)$ had received oxaliplatin, LV and 5-FU (FOLFOX regimen), and $6(25 \%)$ had received taxane (paclitaxel or docetaxel) with cisplatin.

Treatment and outcomes. A total of 113 cycles were conducted, with a median of 4 cycles per patient (range, 1-12 cycles). However, 4 patients who demonstrated early disease progression and 3 patients who demonstratated clinical impairment or toxicity received only 1 or 2 cycles of chemotherapy.

Of the 24 patients, 3 achieved PR and 8 demonstrated $\mathrm{SD}$. On an intent-to-treat basis, the overall RR was $12.5 \%$ and the disease control rate (PR and SD) was $45.8 \%$. The median TTP was 2 months [95\% confidence interval (CI), 1.89-2.11 months] (Fig. 1), the median OS was 5.4 months (95\% CI, 4.11-6.69 months) and the 1-year survival rate was $8.3 \%$ (Fig. 2).

Toxicities. All 24 patients were evaluable for toxicity. The toxicity profiles are shown in Table II. A total of $6(25 \%)$ patients delayed the next cycle of chemotherapy, primarily due to myelosuppression, and 5 (20.8\%) patients required dose reduction due to grade 3-4 toxicities. Grade 3-4 hematological toxicities, including neutropenia, anemia and thrombocytopenia, were observed in $6(25 \%), 4(16.7 \%)$ and $1(4.2 \%)$ patients, respectively. Additionally, 3 (12.5\%) patients developed febrile neutropenia, of which 1 succumbed to pneumonia. 
Table I. Characteristics of 24 frail or elderly patients with advanced gastric cancer.

\begin{tabular}{|c|c|}
\hline Characteristics & No. of patients $(\%)$ \\
\hline \multicolumn{2}{|l|}{ Age (years) } \\
\hline$\geq 65$ & $11(45.8)$ \\
\hline$<65$ & $13(54.2)$ \\
\hline Median & 60.5 \\
\hline Range & $30-83$ \\
\hline \multicolumn{2}{|l|}{ Gender } \\
\hline Male & $17(70.8)$ \\
\hline Female & $7(29.2)$ \\
\hline \multicolumn{2}{|l|}{ ECOG PS } \\
\hline 1 & $6(25.0)$ \\
\hline 2 & $18(75.0)$ \\
\hline \multicolumn{2}{|l|}{ Histology } \\
\hline Adenocarcinoma differentiation & $21(87.5)$ \\
\hline Well & $6(25.0)$ \\
\hline Moderate & $4(16.7)$ \\
\hline Poor & $11(45.8)$ \\
\hline Signet ring cell carcinoma & $3(12.5)$ \\
\hline \multicolumn{2}{|l|}{ Disease status } \\
\hline Recurrent & $7(29.2)$ \\
\hline Metastatic & $17(70.8)$ \\
\hline \multicolumn{2}{|l|}{ Sites of metastases } \\
\hline Liver & $10(41.6)$ \\
\hline Abdominal lymph node & $12(50.0)$ \\
\hline Lung & $4(16.7)$ \\
\hline Peritoneum & $13(54.2)$ \\
\hline Ovary & $2(8.3)$ \\
\hline Bone & $3(12.5)$ \\
\hline \multicolumn{2}{|l|}{ First-line chemotherapy } \\
\hline Oxaliplatin + LV + 5-FU & $14(58.3)$ \\
\hline Taxane and cisplatin & $6(25.0)$ \\
\hline S-1 +/- cisplatin & $4(16.7)$ \\
\hline
\end{tabular}

ECOG PS, European Clinical Oncology Group performance status; $\mathrm{LV}$, leucovorin; 5-FU, 5-fluorouracil.

Non-hematological toxicities were generally mild. Grade 1-2 nausea, vomiting and diarrhea were observed in $10(41.7 \%), 7(29.2 \%)$ and $4(16.7 \%)$ patients, respectively. Grade 3-4 gastrointestinal toxicities, including nausea, vomiting, diarrhea and mucositis, were observed in $3(12.5 \%)$, $2(8.3 \%), 1(4.2 \%)$ and $1(4.2 \%)$ patients, respectively.

\section{Discussion}

Over the past decade, new chemotherapeutic agents, including docetaxel, paclitaxel, capecitabine, S-1, irinotecan and oxaliplatin, have been extensively studied for use in gastric cancer therapy. Although AGC is considered to be chemosensitive, its prognosis has remained poor, with a median OS of approximately 10 months. Gastric cancer develops more frequently
Table II. Toxicity profile by grade of 24 frail or elderly patients with advanced gastric cancer.

\begin{tabular}{lcc}
\hline Toxicity & $\begin{array}{c}\text { Grade 1-2 } \\
(\%)\end{array}$ & $\begin{array}{c}\text { Grade 3-4 } \\
(\%)\end{array}$ \\
\hline $\begin{array}{l}\text { Hematological } \\
\text { Neutropenia }\end{array}$ & $9(37.5)$ & $6(25.0)$ \\
Febrile neutropenia & - & $3^{\mathrm{a}}(12.5)$ \\
Anemia & $18(75.0)$ & $4(16.7)$ \\
Thrombocytopenia & $6(25.0)$ & $1(4.2)$ \\
Non-hematological & & $3(12.5)$ \\
Nausea & $10(41.7)$ & $2(8.3)$ \\
Vomiting & $7(29.2)$ & $1(4.2)$ \\
Diarrhea & $4(16.7)$ & $1(4.2)$ \\
Mucositis & $4(16.7)$ & 0 \\
Hepatotoxity & $5(20.8)$ & \\
\hline
\end{tabular}

${ }^{\mathrm{a}} 1$ case of mortality due to pneumonia.

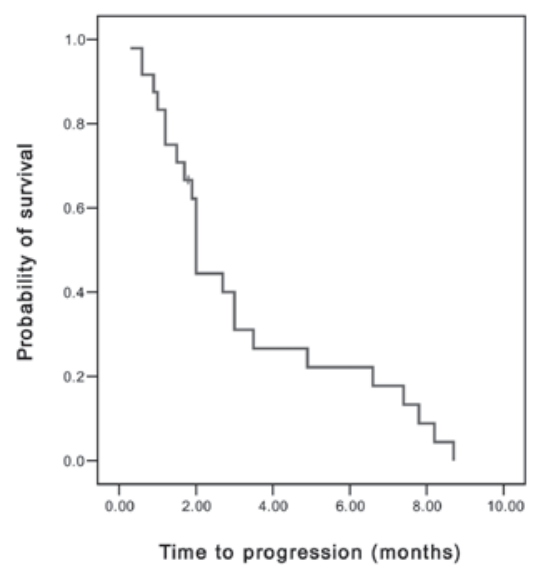

Figure 1. Time to progression of advanced gastric cancer patients, determined from the first day of chemotherapy using the modified FOLFIRI regimen until tumor progression or mortality. FOLFIRI, irinotecan with infusional leucovorin and 5-fluorouracil.



Figure 2. Overall survival of advanced gastic cancer patients, determined from the first day of chemotherapy using the modified FOLFIRI regimen until the date of mortality or the last follow-up. FOLFIRI, irinotecan with infusional leucovorin and 5-fluorouracil. 
among elderly patients (1). Additionally, a considerable number of patients with AGC have poor PS in association with a previous radical gastrectomy or comorbidity; therefore, attempts to develop optimal regimens for frail or elderly patients are required.

The combination of irinotecan with 5-FU/LV (FOLFIRI) as first-line chemotherapy has demonstrated an acceptable safety profile with beneficial activity for AGC patients $(7,8)$. A variety of FOLFIRI regimens have been examined in the clinical setting (9-11); however, in this study we used the modified FOLFIRI regimen (biweekly irinotecan $150 \mathrm{mg} / \mathrm{m}^{2}$ with $\mathrm{LV} 100 \mathrm{mg} / \mathrm{m}^{2}$ and 5-FU 2,000 $\mathrm{mg} / \mathrm{m}^{2}$ ) for patients with AGC We adopted an infusional 5-FU regimen due to low hematological toxicity levels observed compared to bolus 5-FU (12).

Several phase II studies have demonstrated the activity and tolerability of FOLFIRI regimens as salvage chemotherapy (9-11); however, information regarding frail or elderly patients with AGC remains limited. In this study, we reviewed the efficacy and safety of the modified FOLFIRI regimen as salvage chemotherapy in AGC patients with old age or poor PS. On an intent-to-treat basis, the overall RR was $12.5 \%$, with a median TTP of 2 months and a median OS of 5.4 months, which are comparable results to those in other studies using FOLFIRI regimens as salvage treatment $(10,11)$. Jeon et al studied another FOLFIRI regimen (biweekly irinotecan $150 \mathrm{mg} / \mathrm{m}^{2}$ with LV $20 \mathrm{mg} / \mathrm{m}^{2}$ and bolus 5 -FU $400 \mathrm{mg} / \mathrm{m}^{2}$ followed by 5 -FU $600 \mathrm{~g} / \mathrm{m}^{2}$ as a $22 \mathrm{~h}$ infusion on day 1 and 2) as second-line chemotherapy in AGC patients who were previously unsuccessfully treated with the modified FOLFOX-4 regimen (10). In this study, approximately $40 \%$ of patients demonstrated a poor PS, with an RR of $9.4 \%$ and a median TTP and OS of 2 months and 5.84 months, respectively (10). Sym et al have also reviewed various FOLFIRI regimens in AGC patients previously treated with fluoropyrimidine, platinum and taxane (11). Among 131 patients, 12 (12.3\%) demonstrated a tumor response, with a median TTP of 2.2 months and a median OS of 6.2 months (11).

With regards to toxicity, the modified FOLFIRI regimen used in the current study was acceptable. A total of 6 patients delayed their next cycle of chemotherapy due to myelosuppression and 5 required dose reduction due to grade 3-4 toxicities. Although there was 1 treatment-related mortality, hematological toxicities were manageable. The incidence of grade 3-4 myelotoxicities in our study was similar to those reported in other studies that employed the same drugs $(10,11)$. The most common non-hematological toxicities, nausea and vomiting, were observed in 54.2 and $37.5 \%$ of patients, respectively. Our results achieved in frail or elderly patients are encouraging in terms of the efficacy and safety. However, considering that 7 patients terminated chemotherapy of FOLFIRI after only 1 or 2 cycles due to early disease progression, clinical impairment or toxicity, further trials are required to identify the patients most likely to benefit from salvage chemotherapy.

In conclusion, in our study, the modified FOLFIRI regimen used as salvage chemotherapy was effective and acceptable for frail or elderly patients with AGC. Our results suggest that this regimen may be an effective option for these patients.

\section{References}

1. Jung KW, Park S, Kong HJ, et al: Cancer statistics in Korea: incidence, mortality, survival, and prevalence in 2008. Cancer Res Treat 43: 1-11, 2011.

2. Greenlee RT, Murray T, Bolden S and Wingo PA: Cancer statistics, 2000. CA Cancer J Clin 50: 7-33, 2000.

3. Gunderson LL and Sosin H: Adenocarcinoma of the stomach: areas of failure in a re-operation series (second or symptomatic look) clinicopathologic correlation and implications for adjuvant therapy. Int J Radiat Oncol Biol Phys 8: 1-11, 1982.

4. Catalano V, Labianca R, Beretta GD, Gatta G, de Braud F and Van Cutsem E: Gastric cancer. Crit Rev Oncol Hematol 54: 209-241, 2005.

5. Thuss-Patience PC, Kretzschmar A, Bichev D, et al: Survival advantage for irinotecan versus best supportive care as second-line therapy in gastric cancer: a randomized phase III study of the Arbeitsgemeinschaft Internistiche Onkolgie (AIO). Eur J Cancer 47: 2306-2314, 2011.

6. Saltz LB, Cox JV, Blanke C, et al: Irinotecan plus fluorouracil and leucovorin for metastatic colorectal cancer. N Engl J Med 343: 905-914, 2004

7. Kim BG, Oh SY, Kwon HC, et al: A phase II study of irinotecan with biweekly, low dose leucovorin and bolus and continuous infusion 5-fluorouracil (modified FOLFIRI) as first line therapy for patients with recurrent or metastatic gastric cancer. Am J Clin Oncol 33: 246-250, 2010.

8. Bouche O, Raoul JL, Bonnetain F, et al: Randomized multicenter phase II trial of a biweekly regimen of fluorouracil and leucovorin (LV5FU2), LV5FU2 plus cisplatin, or LV5FU2 plus irinotecan in patients with previously untreated metastatic gastric cancer: a Federation Francophone de Cancerologie Digestive Group Study - FFCD 9803. J Clin Oncol 22: 4319-4328, 2004.

9. Kim SG, Oh SY, Kwon HC, et al: A phase II study of irinotecan with bi-weekly, low-dose leucovorin and bolus and continuous infusion 5-fluorouracil (modified FOLFIRI) as salvage therapy for patients with advanced or metastatic gastric cancer. Jpn J Clin Oncol 37: 744-749, 2007.

10. Jeon EK, Hong SH, Kim TH, et al: Modified FOLFIRI as second-line chemotherapy after failure of modified FOLFOX-4 in advanced gastric cancer. Cancer Res Treat 43: 148-153, 2011.

11. Sym SJ, Ryu MH, Lee JL, et al: Salvage chemotherapy with biweekly irinotecan, plus 5-fluorouracil and leucovorin in patients with advanced gastric cancer previously treated with fluoropyrimidine, platinum, and taxane. Am J Clin Oncol 31: 151-156, 2008.

12. Diaz-Rubio E: New chemotherapeutic advances in pancreatic, colorectal, and gastric cancers. Oncologist 9: 282-294, 2004. 